\title{
Esophageal Invasion by an Upper Gastric Cancer: Retrospective Evaluation and Prognosis
}

\author{
JINRYO TAKEDA, KEN HASHIMOTO, KIKUO KOUFUJI, TAKAHO TANAKA, \\ TOURU UMEZU, KENJI YASUMOTO AND TERUO KAKEGAWA
}

\author{
Department of Surgery, Kurume University School of Medicine, \\ Kurume, 830 Japan
}

Received for publication May 11, 1989

\begin{abstract}
Summary: From 1975 to 1984, 894 patients with gastric cancer were operated on in the Department of Surgery at Kurume University School of Medicine. Of these, $86(10 \%)$ had upper gastric cancer invading the esophagus. The 86 resected tumors were divided into two groups according to the surgical approach, thoraco-abdominal or abdominal. Twenty-three (27\%) were resected through the abdominal approach and $63(73 \%)$ were resected by the thoracoabdominal approach. The overall positive mediastinal lymphnode metastasis rates for the 55 patients who underwent mediastinal lymphnode dissection by the thoraco-abdominal approach were $22 \%$ for differentiated type and $37 \%$ for undifferentiated type. The positive mediastinal lymphnode metastasis and the correlation to the extent of esophageal cancer invasion were observed at a minimal $7 \mathrm{~mm}$ distance from the esophago-gastric (E-G) junction for the undifferentiated type, and $2.2 \mathrm{~cm}$ for the differentiated type. Sixteen patients with cancer invading the esophagus were radically resected by the abdominal approach with an overall survival rate of $39 \%$, while 48 were resected by the thoraco-abdominal approach with a 5 year survival rate of $40 \%$. The 86 resected tumors were further subdivided into two groups according to the year of surgery. From 1975 to 1979 , the abdominal approach was employed in $36 \%$ and the thoraco-abdominal approach in $64 \%$ of the surgeries, with an overall curative resection rate of $47 \%$. However, from 1980 to 1984 , the abdominal approach was employed in only $17 \%$ and the thoraco-abdominal approach in $83 \%$ of the surgeries and the curative resection rate increased to $59 \%$. The 5 year survival rate in stage II and III was 39\% for the thoraco-abdominal approach, and $32 \%$ for the abdominal approach. The thoraco-abdominal approach was adopted in those patients with more extensive esophageal involvement or with large tumors. The conclusion of this report is a recommendation of extended distal esophagectomy with total gastrectomy by the thoraco-abdominal approach as the best treatment for upper gastric cancer invading the esophagus.
\end{abstract}

Key words: upper gastric cancer - esophageal invasion - thoraco-abdominal approach - mediastinal lymphnode metastasis - prognosis

\section{Introduction}

The prognosis of upper gastric cancer including cardia cancer is generally poorer than for adenocarcinoma in the corpus or distal stomach, despite more agressive surgical resection. This can be attributed to the difficulty in detecting the cancer early in this area, resulting in a high proportion of advanced cancers with con- 
comitant complex distribution patterns of lymphnode metastasis, involving both mediastinal and abdominal lesions.

A retrospective study of 86 patients, whose upper gastric cancers invading the esophagus were resected with either a thoraco-abdominal or abdominal approach, was made to assess the factors contributing to prognosis for the patients.

\section{Patients and Methods}

From 1975 to 1984, 894 patients with gastric cancer were operated in the Department of Surgery at Kurume University School of Medicine. Of these, 86 (10\%) had upper gastric cancer invading the esophagus, histopathologically. Adenocarcinomas extending to the middle third of the stomach, squamous cell carcinomas and leiomyosarcomas were not included in this investigation. The 86 patients were divided into two groups according to the year of the surgery, 1975 to 1979 and 1980 to 1984 . These were further subdivided according to the surgical approach, thoraco-abdominal or abdominal. The 86 resections were also classified by stage as

TABLE 1

Stage of Carcinoma

\begin{tabular}{c|c|c|c|c|c}
\hline Stage & I & II & III & IV & Total \\
\hline $\begin{array}{c}\text { No. of Cases } \\
(\%)\end{array}$ & 8 & 8 & 45 & 25 & 86 \\
$(9.3)$ & $(9.3)$ & $(52.3)$ & $(29.1)$ & $(100)$ \\
\hline
\end{tabular}

described by the Japanese Research Society for Gastric Cancer in 1981. The number of tumors at each stage are summarized in Table $1.9 \%$ were in stage I, $9 \%$ in stage II, $52 \%$ in stage III and $29 \%$ in stage IV.

\section{Results}

Of the 86 cases involving upper gastric cancer invading the esophagus, 23 (27\%) were resected with the abdominal approach. The remaining $63(73 \%)$ were resected by the thoraco-abdominal approach. Positive mediastinal lymphnode metastasis had occurred in 55 patients who underwent mediastinal lymphnode dissection with the thoraco-abdominal approach, $22 \%$ of these were the differentiated type and $37 \%$ were the undifferentiated type according to Nakamura's classification (1967).

The relationship between positive mediastinal lymphnode metastasis and the extent of esophageal cancer invasion is shown in Table 2. The metastasis was observed at a minimum of $7 \mathrm{~mm}$ from the esophago-gastric (E-G) junction for the undifferentiated type, and $2.2 \mathrm{~cm}$ for the differentiated type.

Sixteen patients with cancer invading the esophagus that were radically resected by the abdominal approach had an overall survival rate of $39 \%$, while 48 that were radically resected by the thoraco-abdominal approach had a 5 -year survival rate of $40 \%$ (Fig. 1).

TABLE 2

Mediastinal Lymphnode Metastasis and Extent of Esophagial Cancer Invasion

\begin{tabular}{l|r|r|r|r|r|r|r}
\hline Length & $\sim 1 \mathrm{~cm}$ & $\sim 2 \mathrm{~cm}$ & $\sim 3 \mathrm{~cm}$ & $\sim 4 \mathrm{~cm}$ & $\sim 5 \mathrm{~cm}$ & $5.1 \mathrm{~cm} \sim$ & Total \\
\hline Histophathology & $0 / 5$ & $0 / 9$ & $2 / 5$ & $3 / 7$ & $1 / 2$ & $0 / 0$ & $6 / 28(21.4 \%)$ \\
Differentiated $(\mathrm{n}=28)$ & $1 / 6$ & $1 / 7$ & $1 / 3$ & $2 / 5$ & $1 / 1$ & $4 / 5$ & $10 / 27(37.0 \%)$ \\
Undifferentiated $(\mathrm{n}=27)$ & $1 / 27$ \\
\hline
\end{tabular}




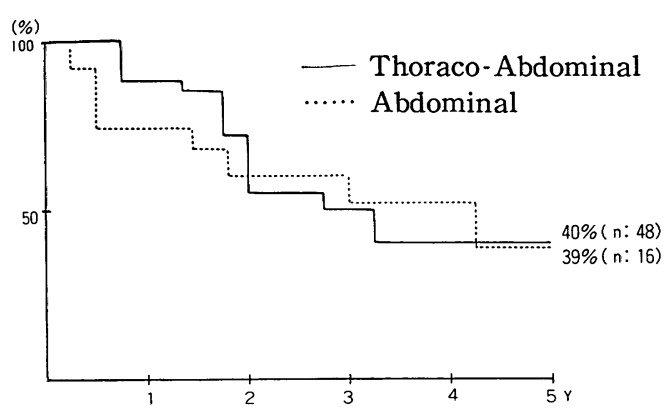

Fig. 1. 5-Years Survival Rate of Gastric Cancer Invading the Esophagus According to Approach - Curative Resection -

TABLE 3

Surgical Approach and Curability

\begin{tabular}{c|c|c|l}
\hline \multirow{2}{*}{ Period } & \multicolumn{2}{|c|}{ Approach } & \multirow{2}{*}{$\begin{array}{l}\text { Curability } \\
\text { Rate }\end{array}$} \\
\cline { 2 - 4 } & Abdominal & $\begin{array}{l}\text { Thoraco- } \\
\text { Abdominal }\end{array}$ & \\
\hline $1975 \sim 1979$ & $35.6 \%$ & $64.4 \%$ & $46.7 \%$ \\
$1980 \sim 1984$ & $17.1 \%$ & $82.9 \%$ & $58.5 \%$ \\
\hline
\end{tabular}

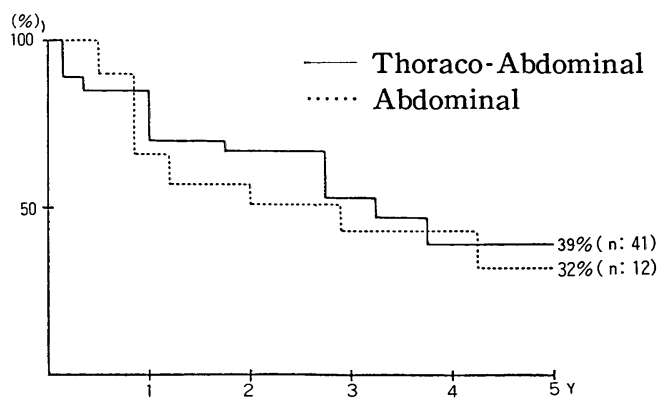

Fig. 2. 5-Years Survival Rate According to Approach in stage II and III - Curative Resection -

In the period from 1975 to 1979 , the abdominal approach was employed in $36 \%$ of the cases and the thoraco-abdominal approach in $64 \%$, with an overall curative rate of $47 \%$. However, in the period from 1980 to 1984 , the abdominal approach was employed in only $17 \%$ of the cases and the thoraco-abdominal approach in $83 \%$ with an overall curative rate of $59 \%$ (Table 3). The thoraco-abdominal approach had a higher curative rate because this approach allows for better resection, reducing the risk of positive cancer cells at the esophageal proximal margin and unsuring complete lymphnode dissection both in the mediastinal and abdominal areas.

The 5 -year survival rate in stage II and III was $39 \%$ by the thoraco-abdominal approach, and only $32 \%$ by the abdominal approach (Fig. 2). While this difference was not significant, the thoraco-abdominal approach was used in patients with more extensive esophageal involvement and with large tumors, demonstrating an advantage of the thoraco-abdominal approach.

\section{Discussion}

The operability and resectability rates for gastric cancer have increased considerably in the past few years. The prognosis for gastric cancer has also improved. How ever, the prognosis for upper gastric cancer is still poorer than for cancer in the corpus or distal stomach despite more aggressive resection for both palliation and cure. Also the risk of surgery has declined markedly due to better preoperative preparation and postoperative care, particularly with regard to the use of hyperalimentation in patients and intensive respiratory therapy. It is difficult to detect cancers in this area, early, which results in a high incidence of advanced cancers and concomitant complex distribution patterns of lymphnode metastasis and infiltration into adjacent organs.

In the present report, 86 resected upper gastric cancers invading the esophagus were reviewed with particular attention to the surgical approach and prognostic factors. Surgery is the only therapy for gastric cancer that can be curative. However, unfortunately, most upper gastric 
cancers frequently spread by direct extension to adjacent organs, including the esophagus, spleen, pancreas, diaphragm, liver and adrenals, and through the gastrocolic ligaments to the transverse colon.

Direct tumor extension into adjacent organs, regional and retroperitoneal lymphnode enlargement, the absence of ascites, and the extent of any liver metastasis should be clearly determined using computed tomograpy (CT) and ultrasonography (US) before an operation.

For cancer in the proximal stomach that has invaded the esophagus, there is no standard surgical therapy. For cancer of the gastric cardia that has invaded the distal esophagus, abdominal, abdominal plus sternotomy, abdominal and transhiatal esophagectomy without thoractomy, left thoraco-abdominal, right thoraco-abdominal, and right thoraco-abdominal plus sternotomy have each been reported as appropriate therapy. The best results were obtained with a radical total gastrectomy employing the thoraco-abdominal approach. The overall mortality rate with this procedure was only $2 \%$. This operation included a total gastrectomy plus resection of the omentum, spleen, body and tail of the pancreas, perigastric and juxta-regional lymphnodes, and occasionally the transverse colon, the left lobe of the liver, part of the diaphragm or paraaortic lymphnodes. Resection of sufficient esophagus to ensure a disease-free margin is recommended.

When tumor invasion of the diaphragm is suspected, partial combined resection of the diaphragm should be performed. Following extensive resection of the diaphragm, reconstruction is achieved using a latissimus dorsi muscle flap of $17 \mathrm{pa}$ tients with upper advanced gastric cancer invading the esophagus who underwent combined resection of the diaphragm during lower esophagectomy and total gastrectomy by the left thoraco-abdominal approach. 7 (41\%) were confirmed to have invisible intradiaphragmatic metastasis, microscopically.

Patients with advanced cancer in the upper gastric portion treated by total gastrectomy had higher survival rates over those treated with only proximal gastrectomy. Papachristou et al. (1980) have also reported that extended total gastrectomy results in a significantly higher survival rate than proximal subtotal gastrectomy.

Lymph flow was examined in the upper gastric portion of mongrel dogs and rabbits by injection of carbon hydrate 44 (CH44) ink into the gastric wall under laparotomy. When $\mathrm{CH} 44$ was injected into the left cadia, lymph flow was observed in both the left and right cardia lymphnodes. Moreover, lymph flow was observed along the left inferior phrenic artery towards the paraaortic lymphnodes and lymphnodes through the left renal vein. Additionally, flow was observed to the diaphragm and upward to the esophagus in the experimental models of upper gastric cancer.

Therefore, it is necessary to determine the distribution patterns of lymph flow and metastasis to have a curative operation. Until early diagnosis is possible, a more extended operation should be considered. In the present clinical studies, the 5 -year survival rate in stage II and III was $39 \%$ by the thoraco-abdominal approach, and $32 \%$ by the abdominal approach. However, the thoraco-abdominal approach was employed in those patients with more extensive esophageal involvement, or with larger sized tumors.

The conclusion of this repport is a recommendation that extended distal esophagectomy with total gastrectomy, occasionally combined with resection of the diaphragm, by the thoraco-abdominal approach is the best treatment for upper gastric cancer invading the esophagus. 
Acknowledgment: The authors would like to thank Mr. Paul R. Maw for his comments on the manuscript.

\section{References}

Fujita, H., Hashimoto, K., Takeda, J., Machi, J. and KaKEgAwA, T. (1988). Diaphragmatic reconstruction using latissimus dorsi muscle flap following wide resection of the diaphragm combined with esophago-gastrectomy for cardial cancer. Jpn. J. Surg. 18, 480-482. Japanese Research Society for Gastric Cancer (1980). The general rules for the gastric cancer study in surgery and pathology. Jpn. J.
Surg. 11, 127-139.

Nakamura, K., Sugano, H., Takagi, K. and Fuchigami, A. (1967). Histopathological study on early gastric carcinoma of the stomach. Some considerations on the ulcer-cancer by analysis of 144 foci of the superficial spreading carcinomas. Gann. 58, 377-387.

Papachristou, D. N. and Fortner, J. G. (1980). Adenocarcinoma of the gastric cardia. The choice of gastrectomy. Ann. Surg. 192, 5864.

Takeda, J., Hashimoto, K., Machi, J., Hirai, Y. and KaKegawa, T. (1987). Surgical treatment for upper gastric cancers. Kurume Med. J. 34, 1-8. 